\title{
Aquisição de uma Tarefa Temporal (DRL) por Ratos Submetidos a Lesão Seletiva do Giro Denteado
}

\author{
The Acquisition of a Temporal Task (DRL) by Dentate Gyrus-selective \\ Colchicine Lesioned Rats
}

\author{
José Lino Oliveira Bueno*, Valéria Catelli Infantozzi Costa, \\ Gilberto Fernando Xavier, \& Leno Luis Dima \\ Universidade de São Paulo
}

\begin{abstract}
Resumo
A lesão seletiva do giro denteado (DG) reduz a eficiência do desempenho de ratos treinados pré-operatoriamente em um esquema de reforçamento diferencial de baixas taxas (DRL); embora os animais lesados sejam capazes de suprimir a resposta de pressão na barra por determinado intervalo de tempo após a resposta anterior, eles subestimam esse intervalo, resultando em um desempenho menos eficiente. Como os animais tinham recebido treinamento pré-operatório, não ficou claro se a lesão interfere na aquisição da discriminação temporal. Este estudo avaliou o efeito da lesão do DG na aquisição de uma tarefa de DRL-2O s. Ratos foram submetidos à neurocirurgia e então ao treino na tarefa de DRL-20 s. Os resultados mostraram que embora os animais lesados se beneficiem do treinamento na tarefa, sua aquisição não é tão eficiente quanto a exibida pelos animais controle. Os resultados sugerem ainda que a lesão do giro denteado interfere na acuidade da discriminação temporal.
\end{abstract}

Palavras-chave: Discriminação temporal; aquisição DRL; hipocampo; giro denteado.

\begin{abstract}
Previous studies have shown that dentate gyrus damage render rats less efficient than sham-operated controls in the performance of a differential reinforcement of low rates of responding (DRL-20 s) task acquired prior to the lesion; even though the lesioned rats were able to postpone their responses after a previous bar press, they seem to underestimate time relative to sham-operated controls, which interferes with their performance. This study investigated the effects of multiplesite, intradentate, colchicine injections on the acquisition and performance of a DRL-20 s task in rats not exposed to preoperatory training, i.e., trained after the lesion. Results showed that the lesioned rats improved along repetitive training in the DRL-20 s task; however, relative to the sham-operated controls, their acquisition rate was slower and the level of proficiency achieved was poorer, indicating that damage to the dentate gyrus interferes with temporal discrimination.

Keywords: Temporal discrimination; DRL task acquisition; hippocampus; dentate gyrus.
\end{abstract}

A capacidade de antecipar a ocorrência de eventos no espaço e/ou no tempo, com base na identificação de regularidades passadas registradas na memória, é de grande valor adaptativo. Muitos tipos de aprendizagem (Ex. os condicionamentos clássico e operante, bem como, aprendizagem de habilidades motoras e perceptuais) mostram suscetibilidade aos arranjos temporais dos eventos como se os organismos fossem projetados a "fazer a coisa certa na hora certa” (Matell \& Meck, 2000). A capacidade de antecipar riscos e oportunidades deve ter levado a um aumento das chances de sobrevivência; assim, foram selecionadas ao longo da evolução (Kuriyama et al., 2003). Não surpreende, portanto, que espécies pertencentes a grupos taxonômicos bastante distantes, incluindo abelhas, peixes, aves, roedores, golfinhos, macacos e humanos, entre

\footnotetext{
Apoio: CNPq e FAPESP.

* Endereço: Setor de Psicobiologia, Dept ${ }^{\circ}$ Psicologia e Educação, FFCL-RP, USP, Av. dos Bandeirantes, 3900, 14040 901, Ribeirão Preto, SP. Fone: (16)36023697; Fax: (16)6335668. jldobuen@ffclrp.usp.br
}

outras, exibam a habilidade de usar informações temporais, antecipando eventos, e que as propriedades psicofísicas dessa habilidade sejam similares nos diferentes grupos (Matell \& Meck, 2000; Paule et al., 1999).

Há diversas evidências experimentais de que o armazenamento de informações sobre uma dada experiência inclui componentes que se referem ao momento da ocorrência do evento (Gallistel, 1989). A capacidade discriminativa de ratos e pombos em relação à duração dos estímulos, i.e., a aprendizagem sobre intervalos temporais, vem sendo intensivamente investigada (Church \& Broadbent, 1990; Gibbon \& Church, 1990; Meck, 1983; Meck \& Church, 1982; Meck, Church, Wenk, \& Olton, 1987; Olton, 1986; Staddon \& Ettinger, 1989). Estes estudos sugerem a existência de processos de mensuração e arquivamento de informações temporais, bem como a habilidade para contar o número de eventos discretos e, assim, estimar a taxa de ocorrência de eventos (Church \& Broadbent, 1990; Gallistel, 1989). 
Diferentes teorias vêm sendo propostas para explicar os processos subjacentes a essas capacidades temporais (Church \& Broadbent, 1990; O’Keefe \& Nadel, 1978; Rawlins, Winocur, \& Gray, 1983; Lejeune, Cornet, Ferreira, \& Wearden, 1998). Church e Broadbent (1990) propõem um modelo de processamento de informações temporais segundo o qual o organismo possuiria um mecanismo análogo a um relógio - Teoria do Relógio Interno (ver também Matell \& Meck, 2000; Wearden \& Bray, 2001). Por outro lado, alguns autores sugerem que as seqüências dos comportamentos desenvolvidos durante os intervalos temporais constituem-se em um relógio comportamental (Teorias do Relógio Comportamental). Este processo temporal, baseado na idéia do relógio comportamental, eliminaria a necessidade de um componente interno de contagem de tempo (relógio interno) (Lejeune et al., 1998; Rawlins et al., 1983) (ver também Matell \& Meck, 2000). A Teoria do Mapa Cognitivo (O’Keefe \& Nadel, 1978) sustenta que os animais podem aprender sobre as relações espaciais de objetos e relacionar eventos com o contexto espacial de sua ocorrência. Estas informações seriam codificadas em mapas cognitivos e, segundo a proposta dos autores, o mapa espacial seria necessário nas tarefas espaciais e temporais já que os animais discriminariam intervalos temporais por meio da realização de comportamentos desenvolvidos durante tais intervalos.

\section{Tarefas Temporais e o Hipocampo}

Existem evidências do envolvimento do hipocampo em tarefas de controle temporal (Meck, Church, \& Olton, 1984; Rawlins et al., 1983; Richelle \& Lejeune, 1980). A lesão do hipocampo causa um prejuízo no desempenho de ratos submetidos a esquemas de reforçamento, como o reforçamento diferencial de baixas taxas (DRL). Neste procedimento, a resposta de pressão na barra é reforçada se, e somente se, ela segue a resposta precedente por um intervalo de tempo específico; neste caso o animal precisa discriminar o tempo decorrido entre a última pressão da barra e o momento atual (Boitano, Dokla, Misikonis, \& Kaluzynski, 1980; Clark \& Isaacson, 1965; Rawlins et al., 1983; Richelle \& Lejeune, 1980). Rawlins et al. (1983) mostraram que ratos com danos hipocampais, produzidos por aspiração de tecido, apresentam substancial deficiência na aquisição de uma tarefa de DRL-12 s, tanto em termos do número médio de respostas corretas, quanto em termos da proporção de respostas corretas em relação ao total de respostas. Isto é, depois de 25 sessões de treinamento, cada qual com 20 minutos de duração, os animais hipocampais apresentavam um nível de desempenho (em termos do número de respostas corretas) igual aos níveis iniciais. Resultados semelhantes foram descritos por Clark, e Isaacson (1965), utilizando o mesmo tipo de lesão, em um esquema de DRL-20 s. Isto é, enquanto os animais controle apresentavam aproximadamente $30 \%$ de respostas reforçadas depois de 14 sessões com 45 minutos cada, os animais lesados apresentaram apenas entre 5 e $10 \%$ de respostas corretas. Similarmente, Jarrard, e Becker (1977) mostraram que ratos com lesão do hipocampo por aspiração treinados em uma tarefa de DRL-20 s, apresentam menos de $1 \%$ de respostas corretas depois de 10 sessões com 45 minutos cada, enquanto os animais controle atingem uma média ao redor de $8 \%$ de respostas corretas. Finger, Green, Tarkonoff, Mortman, e Andersen (1990) testaram ratos em um esquema de DRL-20 s e de DRL-40 s. Animais com lesões eletrolíticas do hipocampo apresentam significativa deficiência no desempenho da tarefa, não conseguindo atingir o mesmo nível de desempenho dos animais controle, mesmo após 30 sessões de treino. Deve-se ressaltar que esses estudos fizeram uso de técnicas convencionais de indução de lesões que invariavelmente eliminam populações de células, interrompem fibras de passagem, produzem danos no sistema vascular, sendo ainda freqüente que áreas adjacentes à estrutura lesada também sofram danos.

Assim, o uso de lesões seletivas das estruturas que compõem a formação hipocampal pode evitar danos a estruturas extra-hipocampais, permitindo uma avaliação mais acurada do papel do hipocampo no processamento das informações em estudo.

Costa, Bueno, e Xavier (2005) avaliaram - mediante aplicação múltipla e tópica de colchicina - os efeitos da lesão seletiva do giro denteado no desempenho de ratos previamente treinados em uma tarefa que envolve controle temporal. Os animais foram treinados em uma tarefa temporal realizada em uma caixa de Skinner em um esquema de DRL-2O s. Na tarefa temporal (DRL) os animais lesados continuam sendo capazes de espaçar suas respostas por um determinado intervalo; porém, esse tempo é subestimado, ou seja, a pressão na barra ocorre em um tempo menor que o previamente treinado.

Em Costa et al. (2005), o nível de desempenho de todos os animais está em torno de $70 \%$ no final dos treinos pré-lesão. Durante as sessões de teste pós-lesão, o grupo controleoperado apresenta uma elevação desse índice, atingindo, no último bloco de testes, valores em torno de $80 \%$. Por outro lado, o grupo lesado mantém ao longo das sessões de teste pós-lesão um índice de eficiência de $70 \%$, igual, portanto, ao índice que apresentava antes da lesão. Costa (2003) encontrou resultados semelhantes.

Neste caso, pode-se dizer que a principal diferença entre os grupos controle-operado e lesado na fase de testes póslesão se dá pelo fato dos animais normais melhorarem ainda mais a eficiência na tarefa, enquanto os animais lesados permanecem com um desempenho semelhante ao apresentado antes da lesão. Portanto, surge a seguinte questão: 1) ocorre um prejuízo da acuidade da discriminação temporal, de tal forma que os animais simplesmente são incapazes de melhorarem seu desempenho a partir de um determinado patamar? $\mathrm{Ou}, 2$ ) esta diferença na manutenção de desempenho pode estar indicando que a lesão afete a aquisição da discriminação temporal?

Para avaliar essas possibilidades realizamos um experimento envolvendo a indução de danos seletivos no giro denteado, pela aplicação múltipla e tópica de colchicina (ver Xavier, Oliveira-Filho, \& Santos, 1999), antes de 
qualquer treinamento na tarefa de discriminação temporal. Depois da neurocirurgia os animais foram submetidos ao treinamento em esquema de reforçamento diferencial de baixas taxas, com $20 \mathrm{~s}$ de intervalo mínimo entre as respostas. Assim, se a lesão impede a aquisição da discriminação temporal, o desempenho dos animais não deve apresentar alterações ao longo do treinamento, ou seja, os animais lesados apresentarão ao final dos treinos, um nível de desempenho igual aos níveis iniciais. Diferentemente, se a lesão não afeta a aquisição da tarefa, haverá melhora do desempenho ao longo das sessões de treino e essa melhora deverá, ao menos em parte, ser paralela à curva de desempenho exibida pelos animais controle. Além disso, foi realizado um esquema de treinamento repetitivo e prolongado para se avaliar em que extensão o prejuízo usualmente observado após lesões do giro denteado pode ser compensado pelo aumento no número de sessões de treino.

\section{Método}

\section{Sujeitos}

Foram utilizados 17 ratos Wistar, machos, adultos pesando de 250 a $300 \mathrm{~g}$ no início do experimento, provenientes do Biotério Central da USP/Campus Ribeirão Preto. No decorrer do experimento os animais foram mantidos em gaiolas individuais, sob regime de privação parcial de alimento.

\section{Equipamentos}

Foram utilizadas caixas experimentais que possuem na parede esquerda ao lado frontal da caixa, uma barra que se encontra a $7 \mathrm{~cm}$ do piso. Abaixo e à esquerda desta, há uma abertura circular por onde são liberadas pelotas de comida como reforço. Cada caixa experimental localizava-se dentro de uma caixa de madeira ( $55 \times 55 \times 55 \mathrm{~cm})$ para isolamento de ruídos externos. A caixa experimental era ligada por uma interface a um computador que controla o experimento e registra os dados.

\section{Neurocirurgia}

Os 17 animais, subdivididos em um grupo com 9 e outro com 8 animais, foram submetidos a neurocirurgia, sob anestesia. A técnica e procedimento utilizados na cirurgia e lesão do GD foram as mesmas utilizadas por Costa et al. (2005) e Xavier et al. (1999). Os animais foram posicionados em um aparelho estereotáxico com a barra dos incisivos ajustada para estar 3,3 $\mathrm{mm}$ abaixo da linha interauricular. $\mathrm{O}$ osso do crânio sobreposto à região almejada foi desgastado com uma broca odontológica até expor a superfície da duramáter; cuidados especiais foram tomados para evitar danos à superfície cortical. Procedimentos usuais de estereotaxia foram utilizados. As injeções foram realizadas utilizandose uma seringa Hamilton de $5 \mu \mathrm{l}$, com uma micropipeta de vidro estirado (diâmetro externo da ordem de $60 \mu \mathrm{m}$ ) colada na ponta da sua agulha. A micro-seringa foi montada sobre um micro-injetor que permite controlar o volume e fluxo de infusão pelo deslocamento controlado do êmbolo da microseringa a uma velocidade definida. Esse sistema foi montado sobre o aparelho estereotáxico (ver detalhes em Costa et al., 2005; Xavier et al., 1999).

Nos animais submetidos à lesão do giro denteado $(n=8)$ injetou-se $0,06 \mu \mathrm{l}$ de colchicina $(7 \mathrm{mg} / \mathrm{ml})$ dissolvida em tampão fosfato (pH 7,4) em cada um de 9 pontos diferentes em cada hemisfério, tomando-se a superfície cortical como coordenada zero-vertical. A micropipeta foi movimentada verticalmente para baixo lentamente para atravessar a duramáter, sua ponta posicionada no local da injeção e a duramáter lavada cuidadosamente com salina. Após a microinfusão de colchicina (ao fluxo de $0,25 \mu \mathrm{l} / \mathrm{min}$.), a micropipeta foi mantida na mesma posição por mais $60 \mathrm{~s}$ para evitar refluxo pelo trajeto da micropipeta no encéfalo; durante esse período a superfície da dura-máter foi mantida umedecida com salina para evitar danos corticais (grupo lesado). Após as injeções, as bordas da pele excisada foram suturadas e os animais transferidos para suas gaiolas, para recuperação (ver detalhes em Costa et al., 2005; Xavier et al., 1999).

Nove animais foram submetidos aos mesmos procedimentos de neurocirurgia, exceto que tampão fostato foi injetado (grupo controle-operado). Três animais do grupo lesado e dois do grupo controle morreram ao longo do experimento. Portanto, os resultados comportamentais referem-se a 12 sujeitos, sendo cinco animais lesados e sete controle-operados.

O período de recuperação da neurocirurgia durou 20 dias, após os quais se introduziu o esquema de privação alimentar e iniciaram-se as sessões de treino conforme descrito abaixo, para ambos os grupos.

\section{Privação alimentar e manipulação}

O esquema de privação limitava o acesso ao alimento de tal forma a manter o peso dos animais em $95 \%$ do peso ad lib. Os animais foram manipulados todos os dias antes de serem colocados nas caixas experimentais e durante a pesagem dos mesmos, o que ocorria diariamente.

\section{Treino a resposta de pressão na barra e comedouro}

Foi realizada uma sessão na qual a aproximação dos animais ao comedouro e/ou a pressão na barra eram reforçadas por meio da liberação de pelotas de alimento. A sessão tinha a duração de 20 minutos ou então, a realização de 50 respostas de pressão na barra seguidas pela ingestão das pelotas de alimento, o que ocorresse primeiro.

\section{Treino em esquema de reforçamento contínuo - CRF}

Após a fase de treino a resposta de pressão na barra e comedouro, os animais foram submetidos a duas sessões, uma sessão por dia, em um esquema de $\mathrm{CRF}$ - reforçamento contínuo: o animal era colocado na caixa experimental, onde a pressão à barra, a qualquer momento, era seguida de reforço. A sessão, para cada animal, tinha a duração de 20 minutos. 


\section{Aquisição da tarefa de DRL}

Os dois grupos foram treinados na tarefa de DRL esquema de reforçamento diferencial de baixas taxas - em dias seguidos, com interrupção de 2 dias antes de serem reiniciados outros 5 dias de treinos. Em cada sessão o animal era colocado na caixa experimental por 20 minutos. Durante este período, suas respostas de pressão à barra só seriam reforçadas quando espaçadas de no mínimo 20 segundos (DRL-20 s). Respostas com um tempo inferior a $20 \mathrm{~s}$ da última resposta não eram reforçadas e, além disso, o tempo era zerado, i.e., começariam a ser contados novamente os 20 segundos. Esse esquema de treinamento foi executado ao longo de 60 sessões, uma por dia.

\section{Análise histológica}

Ao término dos testes comportamentais os animais foram anestesiados profundamente e perfundidos intracardiacamente com $400 \mathrm{ml}$ de solução de sulfeto tamponada. Após a perfusão, os encéfalos foram removidos da caixa craniana, fixados em Carnoy e processados até sua inclusão final em parafina. Depois de emblocados em pararfina, os cérebros foram fatiados em micrótomo. Uma secção de $20 \mu \mathrm{m}$ de espessura foi recolhida em lâmina histológica a cada 10 secções, e corada com violeta de cresila, para evidenciar os corpúsculos de Nissl. Essas secções coradas permitiram avaliar a ocorrência de perda celular no giro denteado, CA1 e outras estruturas. As lâminas foram analisadas ao microscópio para se avaliar a extensão das lesões produzidas.

\section{Análise dos resultados}

O número de respostas reforçadas, o número total de respostas e os intervalos entre as respostas (IER) foram registrados ao longo de todas as sessões. A partir desses registros calculou-se o Índice de Eficiência (IEF) por sessão (Richelle \& Lejeune, 1980): IEF $(\%)=\left[\left(\mathrm{N}^{\prime}+\sum_{\mathrm{Xi}<\mathrm{T}} \mathrm{Xi} /\right.\right.$ $\mathrm{T}) / \mathrm{N}] \mathrm{x}$ 100; sendo, $\mathrm{N}^{\prime}=$ no. de respostas reforçadas, $\mathrm{N}=$ no. total de respostas, $\mathrm{T}=$ tempo crítico, no caso $\mathrm{T}=20$ segundos, $\mathrm{Xi}=$ duração dos intervalos entre respostas menores que $\mathrm{T}$. $\mathrm{O}$ desempenho máximo possível ocorre quando: $\mathrm{N}^{\prime}=\mathrm{N}$ e $\mathrm{Xi}=0$, portanto, $\mathrm{IEF}(\%)=\left[\left(\mathrm{N}^{\prime}+0\right) / \mathrm{N}\right] \times 100=100 \%$.

Os índices de eficiência (IEF) diários dos (n) sujeitos ao longo das sessões foram usados na análise estatística empregando-se uma Análise de Variância para medidas repetidas (ANOVA), utilizando-se "Grupo" como fator entresujeitos e "Blocos de treinamento" como fator intra-sujeitos. Esta análise permitiu a comparação entre as curvas de desempenho dos grupos controle-operados e lesados ao longo das sessões de treino. O Teste de Newman-Keuls foi utilizado como teste de comparações múltiplas complementar à ANOVA. Foram consideradas diferenças significantes comparações cujos níveis de significância foram iguais ou menores do que 0,05.

\section{Resultados}

\section{Resultados Histológicos}

Na Figura 1 é apresentado um desenho esquemático de uma secção coronal (Bregma=-4,16 mm) de encéfalo de rato onde estão assinalados os campos CA1, CA3 e o giro denteado do hipocampo dorsal (Paxinos \& Watson, 1997).

Os animais submetidos à micro-infusão de colchicina exibiram perda substancial das células granulares do giro denteado, bilateralmente, ao longo de todo o eixo septotemporal, associada a uma pequena perda de células piramidais do campo CA1. Além disso, não houve perda aparente de células do córtex sobreposto ao hipocampo. Estes resultados replicam os descritos por Costa et al. (2005) e Xavier et al. (1999), que utilizaram a mesma abordagem neurocirúrgica.

\section{Resultados Comportamentais}

A Figura 2 mostra os IEF dos grupos controle-operado e lesado ao longo do treinamento na tarefa de DRL-2O s, organizados em 12 blocos de cinco sessões cada. A ANOVA revelou a existência de diferenças significantes na comparação dos IEFs em relação ao fator $\operatorname{Grupo}\left(\mathrm{F}_{1,10}=11,46\right.$; $p<0,01)$, Blocos de treinamento $\left(\mathrm{F}_{11,110}=12,53 ; p<0,01\right) \mathrm{e}$ na interação entre Grupos x Blocos de treinamento $\left(\mathrm{F}_{11,110}=2,17 ; p<0,05\right)$, indicando que embora ambos os grupos tenham exibido melhora de desempenho ao longo das sessões de treino, essa melhora foi mais evidente no grupo controle-operado. De fato, a inspeção da Figura 2 revela que os animais do grupo controle exibem uma melhora substancial de desempenho nos primeiros 3 blocos de treinamento, seguida de uma melhora progressiva ao longo das demais sessões de treinamento; já os animais do grupo lesado exibem uma melhora significativa nos primeiros 3 blocos de treino, estabilizando seu desempenho no patamar atingido ao final do terceiro bloco de treinamento.

Nas comparações entre-sujeitos, o Teste de Newman-Keuls complementar à ANOVA revelou a existência de diferenças significantes entre os grupos a partir do oitavo bloco de treinamento. Já nas comparações intra-sujeitos, o Teste de Newman-Keuls complementar à ANOVA revelou, 1) em relação aos animais controle operados, diferenças significantes entre os escores do bloco 1 e os parâmetros correspondentes de todos os demais blocos, exceto o bloco 2 , e diferenças significantes entre os escores do bloco 2 e os parâmetros correspondentes de todos os blocos a partir do oitavo; 2) em relação aos animais lesados, os escores exibidos nos blocos 1 e 2 não diferem entre si, e ambos diferem significantemente em relação aos parâmetros correspondentes de todos dos demais blocos de treinamento que, por sua vez, não diferem entre si (Figura 2).

A inspeção da Figura 2 mostra que ambos os grupos exibem níveis equivalentes de desempenho no primeiro 


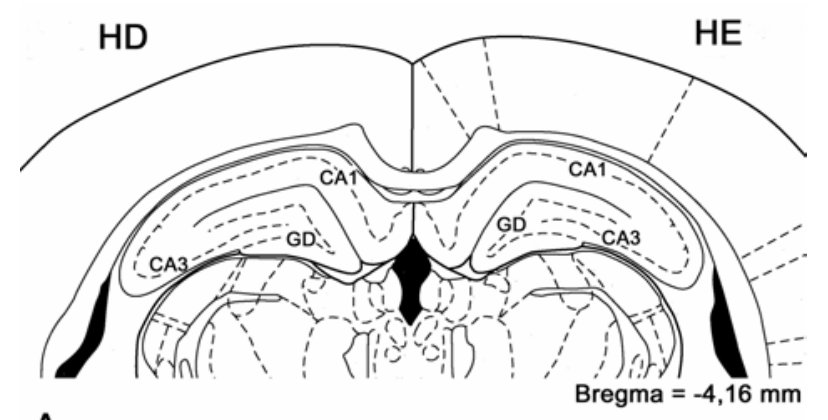

A

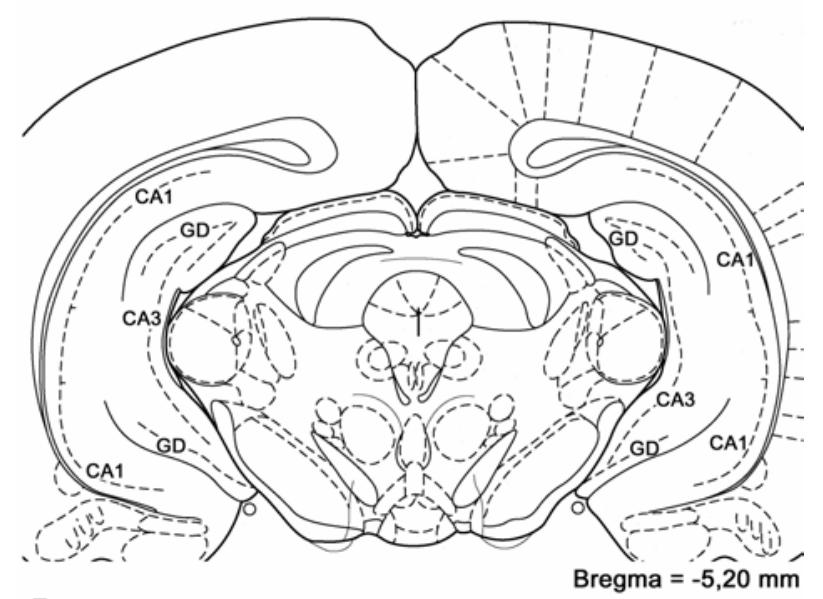

B

Figura 1. Desenhos esquemáticos de seç̧ões coronais dos pólos septal (A) e temporal (B) dos hemisférios direito (HD) e esquerdo (HE) de encéfalo de rato. Assinalados os campos CA1, CA3 e o giro denteado (GD) do hipocampo. Reproduzido a partir de "The Rat brain in Sterotaxic Coordinates", Figuras 34 e 41, de Paxinos, e Watson (1997), com permissão de Elsivier.

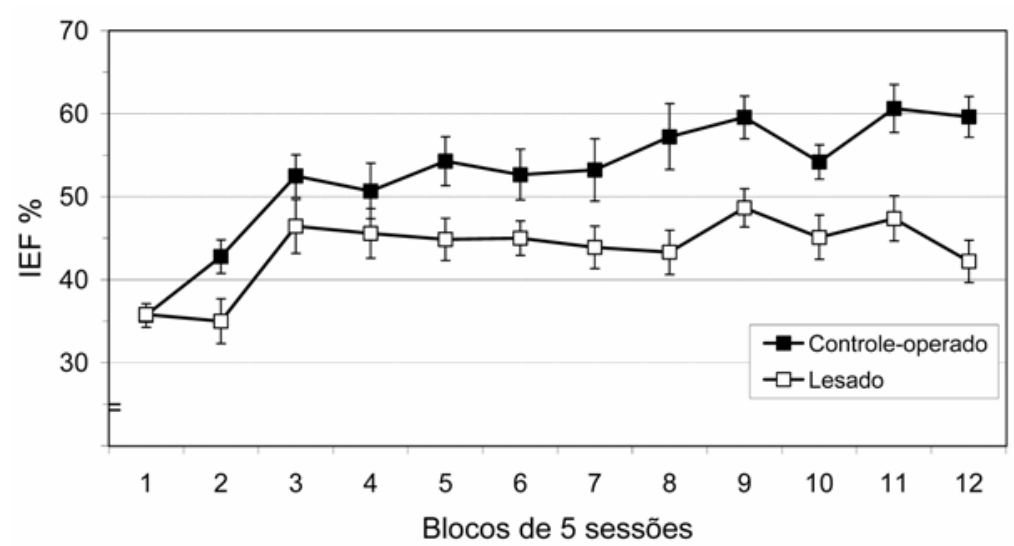

Figura 2. Média ( \pm EP) dos Índices de Eficiência (\%) dos animais controle-operados $(n=7)$ e lesados $(n=5)$ submetidos ao treino de DRL-20 s.

bloco de treinamento (IEFs ao redor de 35\%), mas exibem taxas de aquisição distintas; enquanto o grupo lesado estabiliza seu desempenho a partir do terceiro bloco de treinamento, exibindo IEFs ao redor de $45 \%$, o grupo controle-operado atinge IEFs ao redor de $53 \%$ já no terceiro bloco de treinamento, melhorando seu desempenho com o treinamento repetitivo e atingindo IEFs ao redor de $60 \%$ ao final de 12 blocos de treinamento.
Em síntese, os resultados mostram que 1) os animais controle-operados e os animais lesados apresentam o mesmo nível de desempenho no inicio do treinamento na tarefa; (2) há uma melhora significativa no desempenho dos animais controle ao longo dos blocos, enquanto os animais lesados demoram mais sessões que o grupo controle para apresentar uma melhora no desempenho; e, 3) apesar da melhora no desempenho dos animais lesados, eles não atingem o mesmo nível de desempenho dos animais controle-operados. 


\section{Discussão}

Os resultados do presente experimento mostram que a indução de danos seletivos no giro denteado pela aplicação múltipla e tópica de colchicina promove um prejuízo na aquisição da tarefa de DRL. Diversos trabalhos também mostram que lesões por aspiração ou eletrolíticas do hipocampo e/ou estruturas aferentes ou eferentes provocam um prejuízo na aquisição em tarefas de DRL (Clark \& Isaacson, 1965; Finger et al., 1990; Jarrard \& Becker, 1977; Rawlins et al., 1983; Schmaltz, Wolf, \& Trejo, 1973).

O uso de técnicas convencionais de indução de lesões causa danos extensos, o que dificulta a interpretação dos resultados comportamentais que podem ser severos. Nesse sentido, o uso de neurotoxinas como instrumento para a indução de danos em regiões circunscritas do sistema nervoso certamente representou um avanço, pois permite eliminar parte substancial dos problemas associados às técnicas tradicionais de lesão. No caso do presente estudo, populações individuais de células granulares do GD foram destruídas pela aplicação múltipla e tópica de colchicina, resultando em perda celular significativa restrita à região almejada. Embora os resultados apontem na mesma direção em relação aos estudos envolvendo técnicas convencionais de lesão, as alterações comportamentais observadas são bem mais detalhadas e precisas.

Sinden, Rawlins, Gray, e Jarrard (1986) mostraram que 1) lesão completa do hipocampo; 2) lesão seletiva do campo CA3; e 3) lesão seletiva do subículo, todas pela administração tópica de ácido ibotênico, levam a uma maior lentidão na aquisição de uma tarefa de DRL-12; o aumento no intervalo de tempo da tarefa de DRL de 12 para 18 segundos prejudicou substancialmente o desempenho dos animais com lesão completa do hipocampo mas não o dos animais lesados no subículo ou no CA3, mostrando que apenas danos extensos do hipocampo interferem na capacidade dos animais para mensurar intervalos de tempo.

Costa (2003) e Costa et al. (2005) mostraram que ratos submetidos ao treino pré-operatório na tarefa de DRL-20 s e, posteriormente, à lesão do giro denteado, mantém o nível de desempenho obtido no treino pré-operatório, não exibindo melhora adicional no treino pós-operatório; assim, diferem dos animais controle-operados que exibem melhora adicional de desempenho no treino pós-operatório. No presente estudo investigou-se o efeito da lesão do giro denteado na aquisição da discriminação temporal; isto é, os animais foram submetidos à neurocirurgia para indução de danos seletivos antes de qualquer treinamento na tarefa, evitando-se, assim, a experiência prévia à lesão no desempenho da tarefa.

Os resultados do presente estudo mostraram que a lesão do giro denteado não impede a aquisição da tarefa de DRL20 s. Porém, esse tipo de lesão torna mais lento o processo de aquisição, já que os animais lesados precisaram de maior número de sessões de treino que os animais controle para exibir melhoras no desempenho da tarefa. Além, disso, os resultados sugerem que há um prejuízo na acuidade da discriminação temporal, pois apesar da melhora no desempenho, os animais lesados mantêm IEFs inferiores ao dos animais controle-operados, apesar do treinamento prolongado na tarefa.

Meck et al. (1987, 1984) sugerem que a deficiência no desempenho da DRL apresentada por animais com lesão no hipocampo é devida mais a um prejuízo da memória operacional do que a uma deficiência na habilidade de contar o tempo. A ausência de um estímulo externo para indicar que um intervalo precisa ser contado no procedimento de DRL adiciona a esta tarefa um componente de memória operacional. Para estes autores, a deficiência dos animais lesados na tarefa de DRL decorre do fato destes animais serem incapazes de processar informações na memória operacional. Por outro lado, O’Keefe e Nadel (1978) propõem que animais sejam capazes de resolver tarefas temporais, tais como um esquema de intervalo fixo (FI) e de reforçamento diferencial de baixas taxas (DRL), por meio da utilização de comportamentos colaterais durante o intervalo, como um relógio externo.

Quando seu sistema de mapeamento cognitivo está intacto o uso de comportamentos colaterais como relógio externo pode ser desenvolvido e muito eficientemente propiciar aos animais uma avaliação de intervalos de tempo decorridos. Contudo, o desenvolvimento e a eficiência desses comportamentos tornam-se prejudicados quando o sistema de mapeamento não está disponível devido à lesão hipocampal. Neste caso, os animais precisam desenvolver uma estratégia de guiamento ou orientação egocêntrica para realizarem os comportamentos colaterais adequadamente. Assim, segundo a teoria proposta por Meck et al. (1987, 1984), devido ao prejuízo da memória operacional, os animais lesados não deveriam ser capazes de aprender a tarefa de DRL. Os dados do presente estudo, no entanto, mostraram que os animais lesados foram capazes de adquirir a tarefa, embora com prejuízo de seu desempenho. Por outro lado, a Teoria do Mapa Cognitivo (O’Keefe \& Nadel, 1978) prevê que animais que não tenham seu sistema de mapeamento intacto devido à lesão hipocampal e estejam utilizando-se de uma estratégia de guiamento ou orientação têm dificuldade para resolver tarefas temporais. O uso destas estratégias, então, poderia explicar a maior lentidão dos animais lesados para adquirir a tarefa e seu desempenho inferior ao dos animais controle-operados, mesmo após treinamento prolongado.

\section{Referências}

Boitano, J.J., Dokla, P.M., Misikonis, S., \& Kaluzynski, T. (1980). Effects of Hippocampectomy in an Incremental-Step DRL Paradigm. Physiology \& Behavior, 25, 273-278.

Church, R.M. \& Broadbent, H.A. (1990). Alternative representations of time, number and rate. Cognition, 37, 55-81. Clark, C.V.H. \& Isaacson, R.L. (1965). Effect of Bilateral hippocampal Ablation on DRL Performance. Journal of Comparative and Physiological Psychology, 59, 137-140. 
Costa, V.C.I. (2003). Efeitos de lesões seletivas do giro denteado no desempenho de ratos em tarefas temporais. Tese de Doutorado nãopublicada, Curso de Pós-Graduação em Psicologia Neurociências e Comportamento, Universidade de São Paulo. São Paulo, SP.

Costa, V.C.I., Bueno, J.L.O., \& Xavier, G.F. (2005). Dentate gyrusselective colchicine lesion and performance in temporal and spatial tasks. Behavioural Brain Research, 160, 286-303.

Finger, S., Green, L., Tarnoff, M.E., Mortman, K.D., \& Andersen, A. (1990). Nimopidipine enhances new learning after hippocampal damage. Experimental Neurology, 109, 279-285.

Gallistel, C.R. (1989). Animal Cognition: The Representation of Space, Time and Number. Annual Review Psychology, 40, 155189.

Gibbon. J. \& Church, R.M. (1990). Representation of time. Cognition, 37, 23-54.

Jarrard, L.E., \& Becker, J.T. (1977). The Effects of Selective Hippocampal Lesions on DRL Behavior in Rats. Behavioral Biology, 21, 393-404.

Kuriyama, K., Uchiyama, M., Suzuki, H., Tagaya, H., Ozaki, A., Aritake, S., Kamei, Y., Nishikawa, T., \& Takahashi, K. (2003). Circadian fluctuation of time perception in healthy human subjects. Neuroscience Research, 46, 23-31.

Lejeune, H., Cornet S., Ferreira, M.A., \& Wearden, J.H. (1998). How Do Mongolian Gerbils (Meriones unguiculatus) Pass the Time? Adjunctive Behavior During Temporal Differentiation in Gerbils. Journal of Experimental Psychology: Animal Behavior Processes, 24, 352-368.

Matell, M.S., \& Meck, W.H. (2000). Neuropsychological mechanisms of interval timing behavior. Bioessays, 22, 94-103.

Meck, W.H., \& Church, R.M. (1982). Abstraction of Temporal Attributes. Journal of Experimental Psychology: Animal Behavior Processes, 8, 226-243.

Meck, W.H. (1983). Selective Adjustment of Speed of Internal Clock and Memory Processes. Journal of Experimental Psychology: Animal Behavior Processes, 9, 171-201.

Meck, W.H., Church, R.M., \& Olton, D.S. (1984). Hippocampus, Time, and Memory. Behavioral Neuroscience, 98, 3-22.

Meck, W.H., Church, R.M., Wenk, G.L., \& Olton, D.S. (1987). Nucleus basalis magnocellularis and medial septal area lesions differentially impair temporal memory. Journal of Neuroscience, 7, 3505-3511.
O'Keefe, J. \& Nadel, L. (1978). The Hippocampus as a Cognitive Map. Oxford: Oxford University Press.

Olton, D.S. (1986). Hippocampal Function and Memory for Temporal Context. In R.L. Isaacson \& K.A. Pribran (Eds.), The Hippocampus (pp.281-298). New York: Plenum Press.

Paule, M.G., Meck, W.H., Mcmillan, D.E., Mcclure, G.Y., Bateson, M., Popke, E.J., Chelonis, J.J., \& Hinton, S.C. (1999). The use of timing behaviors in animals and humans to detect drug and/ or toxicant effects. Neurotoxicology and Teratololy, 21, 491-502.

Paxinos, G. \& Watson, C. (1997). The Rat brain in Sterotaxic Coordinates. London: Academic Press.

Rawlins, J.N.P., Winocur, G., \& Gray, J.A. (1983). The Hippocampus, Collateral Behavior, and Timing. Behavioral Neuroscience, 97, 857-872.

Richelle, M. \& Lejeune, H. (1980). Time in Animal Behaviour. Oxford: Pergamon Press.

Schmaltz, L.W., Wolf, B.P., \& Trejo, W.R. (1973). FR, DRL, and discrimination learning in rats following aspiration lesions and penicillin injection into hippocampus. Physiology and Behavior, 11, 17-22.

Sinden, J.D., Rawlins, J.N., Gray, J.A., \& Jarrard, L.E. (1986). Selective cytotoxic lesions of hippocampal formation and DRL performance in rats. Behavioral Neuroscience, 100, 320-329.

Staddon, J.E.R., \& Ettinger, R.H. (1989). Learning: An Introduction to the Principles of Adaptative Behavior. Orlando: Harcourt Brace Jovanovich.

Wearden, J.H., \& Bray, S. (2001). Scalar timing without reference memory? Episodic temporal generalization and bisection in humans. The Quarterly Journal of Experimental Psychology, 54B, 289-309.

Xavier, G.F., Oliveira-Filho, F.J.B., \& Santos, A.M.G. (1999). Dentate gyrus-selective colchicine lesion and disruption of performance in spatial tasks: difficulties in 'place strategy' because of a lack of flexibility in the use of environmental cues? Hippocampus, 9, 668-681. 